\title{
HeartMate XVE malfunction caused by fluid aspiration into the vent port
}

\author{
Sinan A. Simsir, MD, ${ }^{a}$ Shu S. Lin, MD, PhD, ${ }^{a}$ Myra F. Ellis, RN, MSN, ${ }^{a}$ Stuart D. Russell, MD, ${ }^{\text {b }}$ and \\ Carmelo A. Milano, MD, ${ }^{a}$ Durham, NC
}

$\mathrm{T}$ he implantation of a left ventricular assist device (LVAD) provides a clinically meaningful survival benefit and better quality of life compared with medical therapy in patients with advanced heart failure. ${ }^{1}$ Malfunction of the LVAD, however, might significantly reduce the survival benefit it incurs. Seven of 41 deaths in patients with LVADs in the REMATCH trial (Randomized Evaluation of Mechanical Assistance for the Treatment of Congestive Heart Failure) were attributed to device malfunction. ${ }^{1}$ We present the case of a patient who had an unusual device complication. Fluid was aspirated into the vent port, resulting in the malfunction of the device and a clinical emergency. A treatment algorithm for this challenging problem is described.

\section{Clinical Summary}

A 55-year-old man $\left(6^{\prime} 5^{\prime \prime}, 183 \mathrm{lb}\right)$ with idiopathic dilated cardiomyopathy had a HeartMate XVE (Thoratec Corp, Pleasanton, Calif) LVAD system implanted. He was convalescing on a stepdown ward 3 weeks postoperatively, with stable LVAD flows of between 5 and $6 \mathrm{~L} / \mathrm{min}$. The patient was incontinent of urine at 5 AM on the day of the event. Urine was aspirated into the LVAD vent port. The low flow and power limit advisory alarms of the LVAD activated. The low flow persisted, and the patient became hemodynamically unstable. The vent filter was immediately removed, and the patient was placed on his right side, enabling fluid drainage from the vent port. A dry towel was also used to absorb the remaining fluid at the vent port. After several minutes in this position, the alarms stopped, and the LVAD flow returned to previous levels. A new vent filter was placed, and the patient's condition remained stable.

The patient experienced the same malfunction again at 9 AM. The device then stopped, and hand pumping was initiated. Electrical activation was not possible. The patient was systemically anticoagulated. He was transferred to the intensive care unit, and arterial line and pulmonary artery catheters were placed for monitoring. The system controller and power source were removed, and the LVAD was successfully activated with the older pneumatic HeartMate driver. This driver was used with a pressurelimiting device on the pneumatic driveline to avoid injury to the

From the Departments of Surgery ${ }^{\mathrm{a}}$ and Medicine, ${ }^{\mathrm{b}}$ Duke University Medical Center, Durham, NC.

Received for publication Dec 22, 2003; accepted for publication Jan 28, 2004.

Address for reprints: Carmelo A. Milano, MD, Department of Surgery, Box 3043, Duke South, Durham, NC 27710 (E-mail: milan002@mc.duke.edu).

J Thorac Cardiovasc Surg 2004;128:619-21

$0022-5223 / \$ 30.00$

Copyright $\odot 2004$ by The American Association for Thoracic Surgery

doi:10.1016/j.jtcvs.2004.01.038

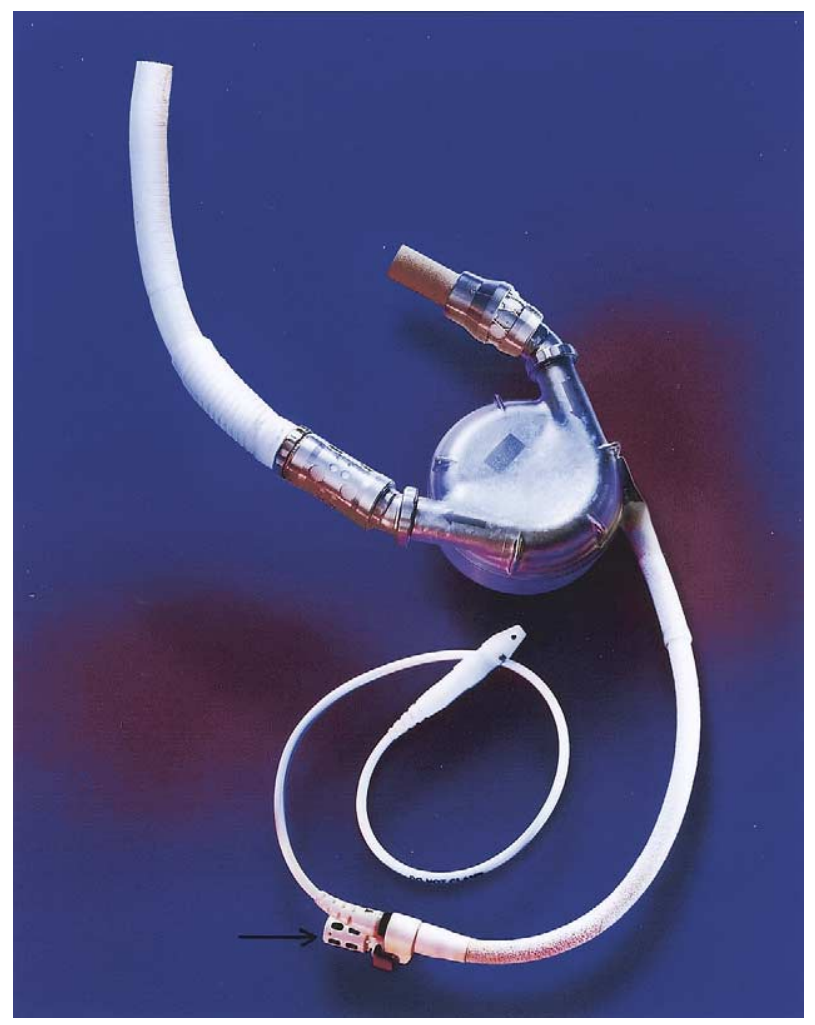

Figure 1. The driveline of the HeartMate XVE LVAD. The arrow points to the vent port.

electric pump, as recommended by the company. The pneumatic driver rate was increased to 90 beats $/ \mathrm{min}$. The patient, however, remained in shock, and respiratory distress ensued. He was mechanically ventilated, and inotropic infusions were initiated to enhance native ventricular function. Despite these maneuvers, the patient was hypotensive, with a central venous pressure of $22 \mathrm{~mm}$ $\mathrm{Hg}$ and a cardiac output of $2.3 \mathrm{~L} / \mathrm{min}$, as measured with the pulmonary artery catheter and the thermodilution method. Metabolic acidosis was present on the arterial blood gases. The arterial pressure tracing suggested poor LVAD stroke volume, and native ventricular ejection confirmed incomplete ventricular unloading by the pneumatically driven HeartMate LVAD.

A decision was made to replace the LVAD surgically at $11 \mathrm{AM}$ given the patient's persistent shock state. At this point, a final attempt was made to reactivate the pump electrically. Fortunately, the pump could be electrically activated and subsequently functioned without alarms. The patient's hemodynamics rapidly normalized: central venous pressure was $8 \mathrm{~mm} \mathrm{Hg}$, and cardiac output increased to $5.3 \mathrm{~L} / \mathrm{min}$. The need to surgically replace the pump was averted. The device continued to function electrically without 


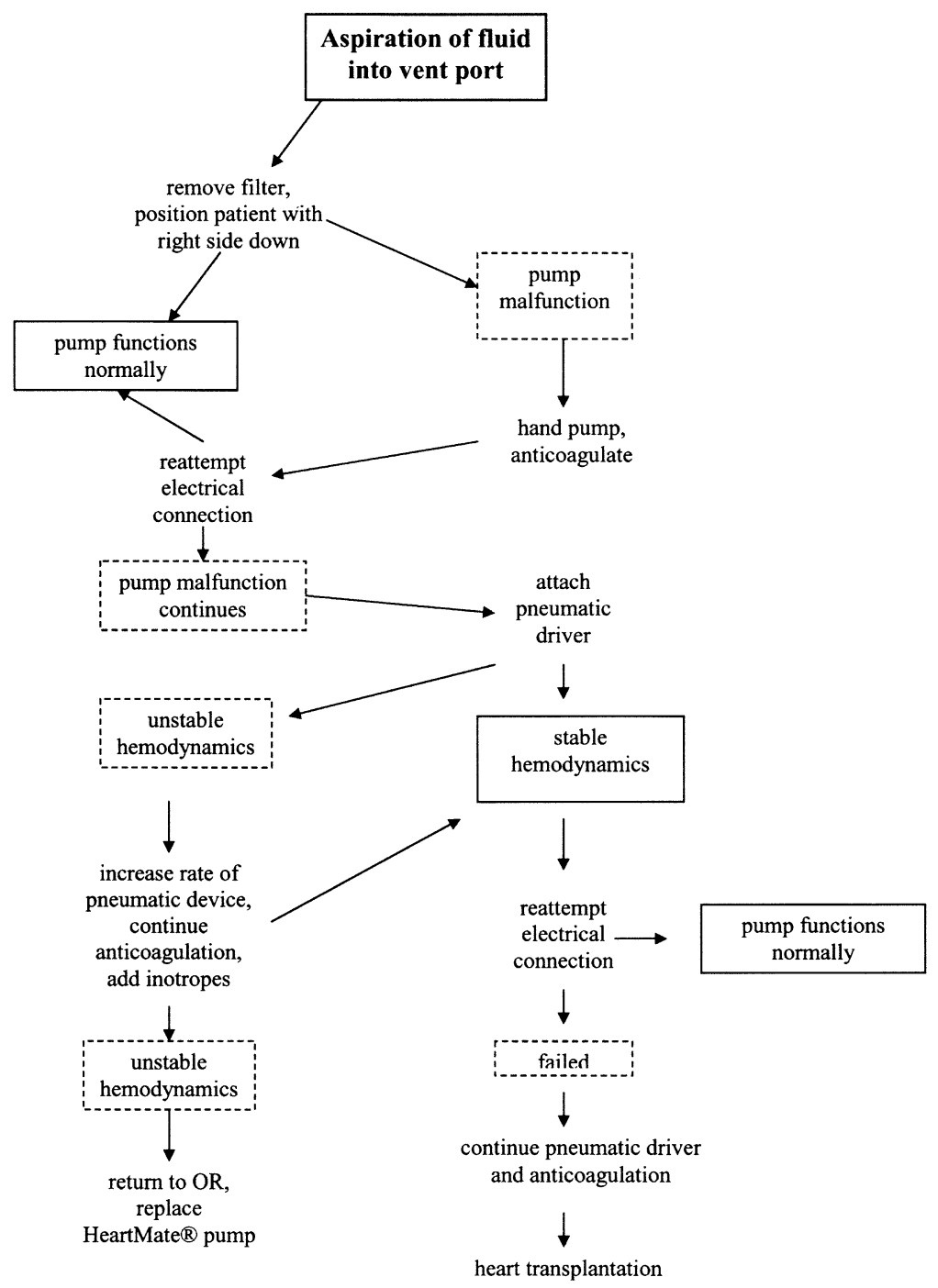

Figure 2. Algorithm for management of fluid aspiration into the vent port of the HeartMate XVE. OR, Operating room.

any further events. The patient is in stable condition with good functional status 3 months postoperatively, having undergone successful cardiac transplantation.

\section{Discussion}

Pusher plate design implanted LVADs use external venting for air, which is displaced as the pump fills and aspirated during pump systole. The HeartMate XVE has a percutaneous driveline, which consists of the electrical lead and the vent port (Figure 1). Particulate matter is prevented from entering the vent port by a filter at the skin. Fluid, however, can pass through the filter into the port and cause device failure, as experienced in our patient.

This case demonstrates several key points. Aspiration of liquid into the vent port can result in shutdown of the device. The inability to electrically drive the pump might be transient or more sustained. Furthermore, device failure might be temporally delayed from the time of the aspiration event. Appropriate maneuvers in this setting appear to include the following: (1) removal of the vent filter; (2) positioning of the patient with the right side down to simply facilitate drainage from the vent port by gravity; (3) attempt to absorb any liquid at the vent port; (4) systemic anticoagulation; (5) hand pumping; (6) attachment to the pneumatic driver; and (7) surgical replacement of the pump.

Notably for this patient, the pneumatic driver failed to achieve adequate hemodynamics. This is explained by the fact that the pump stroke volume is significantly reduced if the electric pump is driven by the old pneumatic driver. Furthermore, excursion of the pusher plate might be compromised after such fluid aspiration into the vent port. In fact, on the basis of the cardiac output and pneumatic driver rate, which was set at 90 beats/min, the pump stroke volume when driven by the pneumatic driver was only 26 $\mathrm{mL}$. Therefore use of the pneumatic driver might only be a short-term or incomplete solution. Furthermore, incomplete emptying of the pump with the pneumatic driver might pose a risk of thromboembolism, emphasizing the need for anticoagulation. Nevertheless, in this case support with the pneumatic driver for 2 hours 
resulted in elimination of vent port obstruction, such that the pump could again function electrically. This averted the need for surgical replacement of the pump. Through this experience, we propose an algorithm, which might help caregivers with this difficult situation (Figure 2).

\section{Reference}

1. Rose EA, Gelijns AC, Moskowitz AJ, Heitjan DF, Stevenson LW, Dembitsky W, et al. Long-term mechanical left ventricular assistance for end-stage heart failure. N Engl J Med. 2001;345:1435-43.

\title{
Surgical management of bilateral multiple invasive pulmonary aspergillosis
}

\author{
Karin M. Dunst, MD, and Ludwig C. Mueller, MD, Innsbruck, Austria
}

$\mathrm{T}$ here are 3 clinical types of pulmonary aspergillosis. The allergic and saprophytic forms can be encountered in immunocompetent patients, whereas invasive pulmonary aspergillosis (IPA) is a life-threatening opportunistic infectious complication that develops in the immunocompromised host as a consequence of impaired defense function. ${ }^{1}$ IPA represents a major source of morbidity and mortality in the neutropenic patient. The clinical symptoms are sometimes masked, especially in the setting of a malignant neoplastic disorder; however, as a rule, there is productive cough and hemoptysis up to life-threatening bleeding. Standard therapy consists of amphotericin B, itraconazole, or both ${ }^{2}$; however, new antifungal agents, such as voriconazole ${ }^{3}$ or caspofungin, ${ }^{4}$ might yield superior therapeutic results. Surgical removal of infected lung tissue is considered as a therapy of last resort. ${ }^{5}$

\section{Clinical Summary}

A 63-year-old woman received high-dose cortisone therapy because of a local seroma complication after surgical removal of a thoracic ependymoma. One month later, she had a septic shocklike syndrome with acute respiratory insufficiency, requiring orotracheal intubation with 100\% oxygen and positive end-expiratory pressure $\left(10 \mathrm{~cm} \mathrm{H}_{2} \mathrm{O}\right)$. This management led to a satisfactory arterial oxygen saturation level (88\%). Bronchoalveolar lavage and sputum cultures revealed Aspergillus fumigatus, and antifungal therapy with amphotericin B was initiated. Thoracic computed tomography showed multiple focal cavitary mycotic lesions, with a cavitary pattern in both of the upper lobes and dense infiltrates in

\footnotetext{
From the Department of Cardiac Surgery, Leopold-Franzens University, Innsbruck, Austria.

Received for publication Jan 6, 2004; revised Jan 31, 2004; accepted for publication Feb 4, 2004.

Address for reprints: Karin M. Dunst, MD, Department of Cardiac Surgery, University Hospital Innsbruck, Anichstrasse 35, 6020 Innsbruck, Austria (E-mail: Karin.dunst@uibk.ac.at).

J Thorac Cardiovasc Surg 2004;128:621-2

$0022-5223 / \$ 30.00$

Copyright (C) 2004 by The American Association for Thoracic Surgery doi:10.1016/j.jtcvs.2004.02.015
}

the lower lobes (Figure 1). Surgical drainage of the largest cavities was undertaken because of failure of conservative management. After 8 weeks of antimycotic treatment, we decided to perform surgical resection. By that

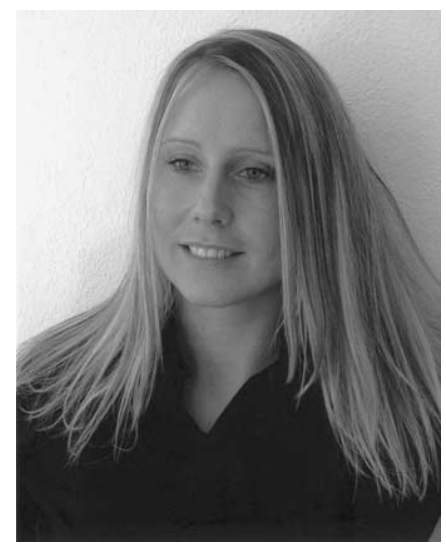

Dr Dunst time, the patient was ventilated through a tracheostoma, with intermittent positive airway pressure. In a first operation, a right upper lobectomy was undertaken (Figure 2). Because of the patient's critical condition, a left upper lobe segmental resection was carried out 4 days later. Furthermore, inflammatory infiltrates in both lungs were omitted from surgical resection. The postoperative course was uneventful, and the patient was discharged from the hospital after 10 days for rehabilitation. At 18 months' followup, the patient has slight exertional dyspnea, and the chest radio-

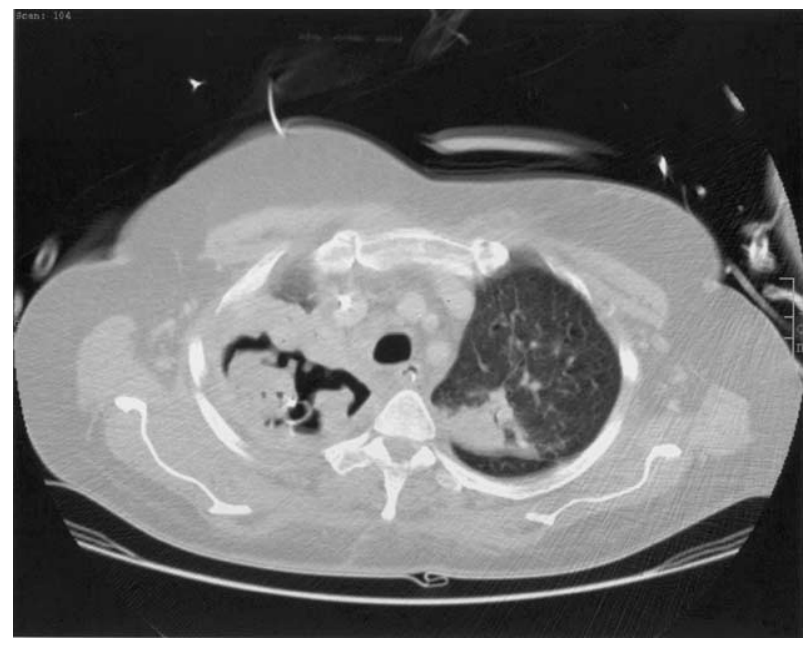

Figure 1. Thoracic computed tomogram showing multiple cavitary lesions with surgical drainage in the right upper lobe. Additionally, there is a consolidated lesion in the left upper lobe, with occasional air trapping. 